D. M. Riffe

\title{
Temperature dependence of silicon carrier effective masses with application to femtosecond reflectivity measurements
}

\author{
D. M. Riffe \\ Department of Physics, Utah State University, Logan, Utah 84322-4415
}

Received August 7, 2001; revised manuscript received October 18, 2001

\begin{abstract}
The conductivity effective masses of electrons and holes in Si are calculated for carrier temperatures from 1 to $3000 \mathrm{~K}$. The temperature dependence of the electron mass is calculated by use of a phenomenological model of conduction-band nonparabolicity that has been fitted to experimental measurements of the dependence of the electron conductivity effective mass on carrier concentration. The hole mass is investigated by tightbinding calculations of the valence bands, which have been adjusted to match experimental values of the valence-band curvature parameters at the top of the valence band. The calculations are in excellent agreement with femtosecond-laser reflectivity measurements of the change in optical effective mass as hot carriers cool from 1550 to $300 \mathrm{~K}$. (c) 2002 Optical Society of America
\end{abstract}

OCIS codes: $160.4760,320.7130,160.6000,320.2250$.

\section{INTRODUCTION}

The conductivity effective masses of electrons $\left(m_{e}\right)$ and of holes $\left(m_{h}\right)$ are important parameters that affect the mobility, electrical resistivity, and free-carrier optical response of a semiconductor. Most commonly, these masses are simply designated by their values at their respective band edges, which for Si are $m_{e 0}=0.259 m_{0}$ and $m_{h 0}=0.33 m_{0}$, where $m_{0}$ is the free-electron mass. However, under conditions of large carrier concentration or elevated temperature, the conductivity masses can vary significantly from their band-edge values. These variations are due to the complicated nature of real bands in a semiconductor, which can include nonparabolicity and the presence of individual bands that are split off from the bands that define the conduction- or valenceband edges. ${ }^{1}$

Ultrafast laser excitation of a semiconductor can easily produce large carrier densities, temperatures, or both, significantly altering the optical response of the free carriers from low excitation values. For example, in a picosecond experiment with $\mathrm{Si}$ in which holes and electrons were excited to a common density of $\sim 7 \times 10^{20} \mathrm{~cm}^{-3}$ at $\sim 1000 \mathrm{~K}$, an optical effective mass $\left[m_{\text {opt }}=\left(1 / m_{e}\right.\right.$ $\left.\left.+1 / m_{h}\right)^{-1}\right]$ approximately $20 \%$ larger than the bandedge value was inferred. ${ }^{2}$

This result, as well as other picosecond optical studies, ${ }^{3-5}$ prompted two theoretical investigations of density-dependent conductivity masses in $\mathrm{Si}$. In the first investigation, based on a pseudopotential-derived bandstructure calculation of $\mathrm{Si}$, the density dependence of the carrier effective masses at 300 and $3000 \mathrm{~K}$ was calculated. ${ }^{6}$ In the second study, based on a simple phenomenological model of the nonparabolicity of the conduction and valence bands, the density dependence at 300 and $1700 \mathrm{~K}$ was calculated. ${ }^{7}$ Whereas the electron conductivity masses determined in the two calculations agree quite well, the calculated density dependences of the hole effective masses are quite different. For example, at a hole concentration of $10^{21} \mathrm{~cm}^{-3}$ and $300 \mathrm{~K}$ the pseudopotential result is $m_{h}=0.95 m_{0}$, whereas the phenomenological calculation yields $m_{h}=0.45 m_{0}$.

In a picosecond investigation of Si the time scale of the measurement is much longer than the electron-phonon energy relaxation time of $\sim 250 \mathrm{fs}^{8}$ Thus those early ultrafast measurements are of carriers that have thermalized with the lattice. With femtosecond probing of Si the situation is entirely different. One now has the possibility of detecting - in real time - the change in optical mass as excited carriers relax to the band edge. Recently I measured the femtosecond time scale reflectivity of Si excited to a carrier density of $\sim 5 \times 10^{18} \mathrm{~cm}^{-3}$ at an initial carrier temperature of $1550 \mathrm{~K}$. These reflectivity measurements are able to follow the carriers as they cool to near room temperature and equilibrate with the lattice. As is discussed in detail below, part of the time dependence of the reflectivity signal is consistent with a significant decrease in the optical mass as the electrons and holes cool to their respective band edges: the experimental results indicate a change in optical mass of $22.9 \pm 0.4 \%$ from 300 to $1550 \mathrm{~K}$.

This precise experimental observation (and the inconsistency in effective masses obtained in the two previous calculations) has prompted an evaluation of the temperature dependence of the conductivity effective masses of electrons and holes in Si. Specifically, we evaluate both $m_{e}\left(n_{e}, T\right)$ and $m_{h}\left(n_{h}, T\right)$ as functions of carrier temperature $T$ from 1 to $3000 \mathrm{~K}$, assuming nondegenerate carrier densities $\left(n_{e}, n_{h}<\sim 10^{20} \mathrm{~cm}^{-3}\right)$. We designate these nondegenerate conductivity masses $m_{e}(T)$ and $m_{h}(T)$, respectively. Our approach in calculating the effective masses is to use the simplest model that is consistent with available experimental data on the conductivity masses. First we calculate $m_{e}(T)$, using a phenomenological description of the conduction-band dispersion 
relation ${ }^{1,9}$ that has been fitted to the density dependence of the electron conductivity mass, which has been determined from room-temperature reflectivity data. ${ }^{10-13}$ For the valence band such a simple phenomenological description is precluded because of the complex shapes of the light-hole and heavy-hole bands that define the valenceband maximum and the presence of the split-off band at $-0.044 \mathrm{eV}$. Thus for $m_{h}(T)$ we must use an accurate k-space description of the valence-band structure. The simplest description of the valence bands that is accurate enough to use in determining $m_{h}(T)$ is provided by tightbinding theory. Here we use an orthogonal, three-center model that includes up to third-neighbor interactions, ${ }^{14}$ which we have extended to explicitly include the spinorbit interaction. ${ }^{15}$ In our calculations we start with three previously derived sets of three-center interaction parameters ${ }^{14,16,17}$ that are slightly modified to exactly reproduce experimental valence-band curvature parameters ${ }^{18-20}$ (which describe the shapes of the three valence bands near their band maxima ${ }^{21}$ ), thus ensuring accurate low-temperature values of $m_{h}(T)$. We then use our calculated values of $m_{e}(T)$ and $m_{h}(T)$ to calculate the temperature-dependent optical effective mass $m_{\text {opt }}(T)$. The result for $m_{\text {opt }}(T)$ is then compared with our measured change in optical mass at 300-1550 K.

\section{ELECTRON EFFECTIVE MASS}

For the Si conduction band the major contribution to the concentration and temperature dependence of $m_{e}\left(n_{e}, T\right)$ is the effect of nonparabolicity in the band that defines the conduction-band minimum. ${ }^{6}$ This nonparabolicity has been inferred experimentally from drift-velocity-, ${ }^{22}$ optical absorption-coefficient-, ${ }^{23}$ and carrier-densitydependent ${ }^{12,13}$ reflectivity measurements. The nonparabolicity is often described by a single parameter $\alpha$ that appears in an effective dispersion relation for the conduction band near each of its six equivalent energy minima ${ }^{1}$ :

$$
E(1+\alpha E)=\frac{\hbar^{2} k^{2}}{2 m_{e 0}} .
$$

Here $E$ is the energy above the band minima and $k$ is the magnitude of the electron wave vector (relative to $\mathbf{k}$ at each of the six minima). For $\mathrm{Si}, \alpha$ is close to $0.5 \mathrm{eV}^{-1}{ }^{22,23}$

For a carrier in state $\mathbf{k}$ with energy $E_{i}(\mathbf{k})$ in a band labeled by subscript $i$, the direction averaged band mass $m_{i}(\mathbf{k})$ associated with that electron is given by ${ }^{24}$

$$
\frac{1}{m_{i}(\mathbf{k})}=\frac{1}{3 \hbar^{2}} \nabla_{k}^{2} E_{i}(\mathbf{k}) .
$$

The conductivity effective mass $m_{c}\left(n_{c}, T\right)$ (where $c$ is either $e$ for electrons or $h$ for holes) is the thermal average of this quantity:

$$
\frac{1}{m_{c}\left(n_{c}, T\right)}=\frac{\sum_{i, \mathbf{k}} f\left[\mu_{c}\left(n_{c}\right), E_{i}(\mathbf{k}), T\right]\left[1 / m_{i}(\mathbf{k})\right]}{\sum_{i, \mathbf{k}} f\left[\mu_{c}\left(n_{c}\right), E_{i}(\mathbf{k}), T\right]} .
$$

Here $f(\mu, E, T)$ is the Fermi-Dirac distribution function and $\mu_{c}\left(n_{c}\right)$ is the chemical potential (i.e., Fermi level), which is simply related to carrier density $n_{c} \cdot{ }^{25}$

Inasmuch as the effective dispersion relation [Eq. (1)] is a function of only the magnitude of $\mathbf{k}$, it is simplest to perform the calculation of $m_{e}\left(n_{e}, T\right)$ in energy space. From Eq. (1), the energy-dependent electron conductivity mass is determined to be

$$
\frac{1}{m_{c}(E)}=\frac{1}{m_{e 0}} \frac{1+(8 / 3) \alpha\left(E+\alpha E^{2}\right)}{\left[1+4 \alpha\left(E+\alpha E^{2}\right)\right]^{3 / 2}} .
$$

For $\alpha E<1$ the energy-dependent correction to the mass is nearly linear relative to $E$, and $m_{e}(E)$ can be expressed, with an accuracy of better than $2 \%$, as

$$
m_{e}(E)=m_{e 0}\left(1+\frac{10}{3} \alpha E\right) .
$$

Also from Eq. (1), the density of electronic states is determined to be

$$
\rho_{i}(E)=\frac{2^{1 / 2} m_{e 0}^{3 / 2}}{\pi^{2} \hbar^{3}} E^{1 / 2}(1+\alpha E)^{1 / 2}(1+2 \alpha E) .
$$

(This is the effective density of states of each of the six individual bands that compose the near-edge part of the conduction band.) With Eqs. (4) and (6) the sums in Eq. (3) can be converted to energy integrals and $m_{e}\left(n_{e}, T\right)$ determined.

I have analyzed reflectivity-derived values of $m_{e}\left(n_{e}, 300 \mathrm{~K}\right)$, plotted in Fig. 1, to extract a value of $\alpha$ for the Si conduction band. The data in Fig. 1 are from four independent measurements of $\mathrm{Si}$ reflectivity. ${ }^{10-13}$ The

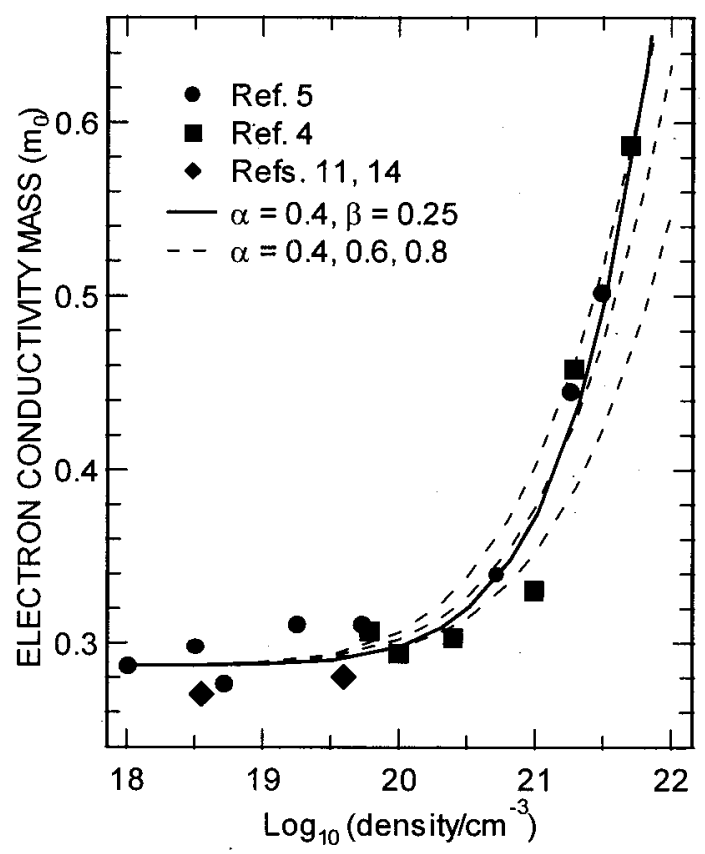

Fig. 1. Si electron conductivity mass versus carrier density at room temperature. Symbols indicate experiment points. Dashed curves, standard model of nonparabolicity, Eq. (1); solid curve, extended model, Eq. (7). For comparison among the calculated curves, $m_{e 0}$ has been slightly adjusted in each calculation such that the low-density, room-temperature mass equals $0.285 m_{0}$. 
three dashed curves shown in the figure were calculated for values of $\alpha=0.4,0.6,0.8 \mathrm{eV}^{-1}$. As is evident from Fig. 1, the highest-density values of $m_{e}\left(n_{e}, 300 \mathrm{~K}\right)$ are consistent with a value of $\alpha=0.8$ (top dashed curve). However, $m_{e}\left(n_{e}, 300 \mathrm{~K}\right)$ values for $n_{e}$ of $10^{20}$ to $10^{21} \mathrm{~cm}^{-3}$ are significantly overestimated by this value of $\alpha$. The data in this density range are better described by $\alpha$ $=0.4 \mathrm{eV}^{-1}$ (lowest dashed curve). As shown by the middle dashed curve, $\alpha=0.6 \mathrm{eV}^{-1}$ best approximates the overall shape of all the reflectivity data.

The value $0.6 \mathrm{eV}^{-1}$ is in good agreement with other experimental determinations. The earliest extracted value comes from drift-velocity measurements, ${ }^{22}$ which were fitted with a value of $0.5 \mathrm{eV}^{-1}$. More recently, optical absorption-coefficient $\operatorname{data}^{23}$ were fitted with $\alpha$ $=0.4 \mathrm{eV}^{-1}$. Previously, reflectivity data (circles in Fig. 1) were also analyzed, ${ }^{13}$ yielding $\alpha=0.27 \mathrm{eV}^{-1}$. However, in that analysis an incorrect formula for the band mass was used [cf. Eq. (2)], resulting in a value of $\alpha$ much lower than we derive from the reflectivity data. As shown in Fig. $1, \alpha=0.6 \mathrm{eV}^{-1}$ does a reasonable job of describing that particular data set, in addition to the other data included in the figure.

In spite of the fairly good agreement in $\alpha$ among the three analyses, the reflectivity data suggest that a higher order of nonparabolicity may be indicated for Si. The effective dispersion relation, Eq. (1), originates from $k \cdot p$ calculations of the Si conduction band structure, ${ }^{1,9}$ where the left-hand side is a Taylor series expansion in the energy $E$. If the next term in the Taylor series is included, the dispersion relation is extended to become

$$
E\left(1+\alpha E+\beta E^{2}\right)=\frac{\hbar^{2} k^{2}}{2 m_{e 0}} .
$$

Using this effective dispersion relation and following the steps as outlined above for calculating $m_{e}\left(n_{e}, 300 \mathrm{~K}\right)$, I also analyzed the data in Fig. 1 with various values of $\alpha$ and $\beta$. As shown by the solid curve in the figure, a somewhat better description of $m_{e}\left(n_{e}, 300 \mathrm{~K}\right)$ at all density values was obtained with $\alpha=0.4 \mathrm{eV}^{-1}$ and $\beta=0.25$ $\mathrm{eV}^{-2}$.

The density dependence of the electron conductivity mass shown in Fig. 1 for $\alpha=0.4 \mathrm{eV}^{-1}$ and $\beta=0.25$ $\mathrm{eV}^{-2}$ is in very good agreement with that determined in the two previous calculations of the electron conductivity mass, ${ }^{6,7}$ one of which was based on a realistic band structure of the conduction band. ${ }^{6}$ Thus, although the phenomenological model used here is simplistic, it accurately describes the effect of nonparabolicity in the conduction band.

Using parameter values extracted from the standard ( $\beta=0 \mathrm{eV}^{-1}$ ) and extended models of nonparabolicity, I calculated $m_{e}(T)$, the nondegenerate limit of $m_{e}\left(n_{e}, T\right)$. In this limit the Fermi-Dirac distribution function is equivalent to a classical Boltzmann distribution for which $f=f(E, T) \propto \exp \left(-E / k_{B} T\right)$, resulting in a mass that is independent of $n_{e}$. For the standard model the temperature-dependent mass is linear in temperature and can be expressed with excellent accuracy up to 3000 $\mathrm{K}$ as

$$
m_{e}(T)=m_{e 0}\left(1+5 \alpha k_{B} T\right) .
$$

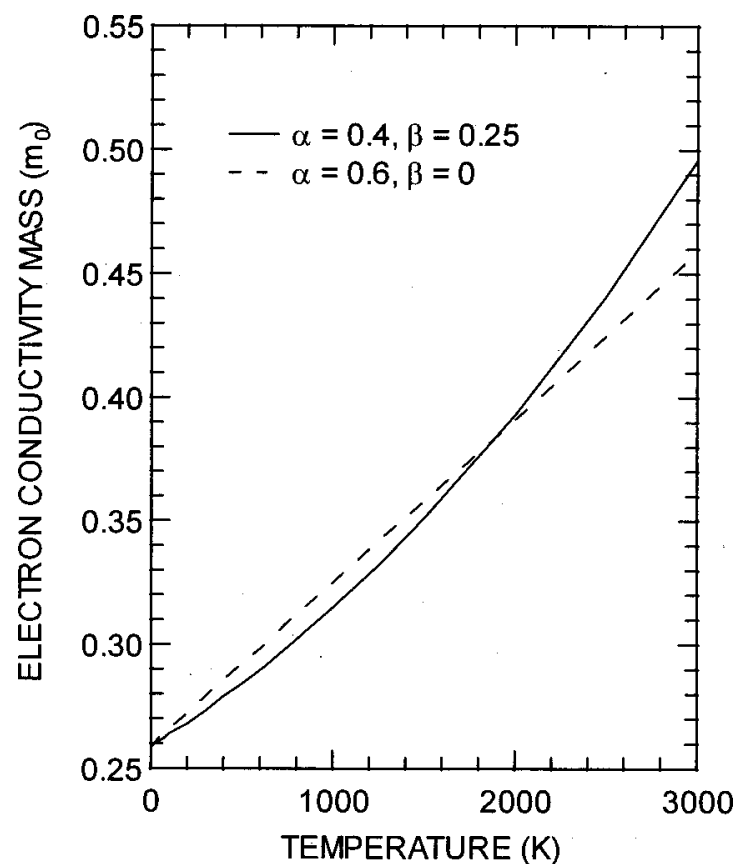

Fig. 2. Calculated electron conductivity mass versus temperature in the nondegenerate carrier-density limit. Dashed curve, standard model of nonparabolicity with $\alpha=0.6 \mathrm{eV}^{-1}$; solid curve, extended model with $\alpha=0.4 \mathrm{eV}^{-1}$ and $\beta=0.25 \mathrm{eV}^{-2}$.

Note that Eq. (8) is simply Eq. (5) with $E$ replaced by $(3 / 2) k_{B} T$. In Fig. $2 m_{e}(T)$ is plotted for $\alpha=0.6 \mathrm{eV}^{-1}$, $\beta=0 \mathrm{eV}^{-2}$ and $\alpha=0.4 \mathrm{eV}^{-1}, \beta=0.25 \mathrm{eV}^{-2}$. The linear variation of $m_{e}(T)$ with $T$ for the standard model is clearly evident. Note that the difference in $m_{e}(T)$ for these two sets of parameters is really significant only at the highest temperatures. Thus, whereas a nonzero value of $\beta$ fits the density-dependent data more precisely, use of the extended model does not dramatically alter the calculated values of $m_{e}(T)$ compared with those of the standard model.

\section{HOLE EFFECTIVE MASS}

The near-band-edge valence-band structure of $\mathrm{Si}$ is much more complicated than that of the conduction band, which leads to a more complex dependence of $m_{h}\left(n_{h}, T\right)$ on $T .{ }^{21}$ The top of the valence band, located at $k=0$, comprises two distinct bands, designated heavy-hole and light-hole bands. Just below the degenerate maxima of these two bands resides the maximum of a third band at $-0.044 \mathrm{eV}$, designated the split-off band. ${ }^{21,26}$ Because of the avoided-crossing nature of the light-hole and split-off bands, the low-energy shape of the light-hole band is particularly complex. At higher (hole) energies a nonparabolicity similar to that observed for the conduction band is expected to dominate the effect of the band structure on $m_{h}\left(n_{h}, T\right){ }^{6,7}$ As is shown below, these effects of avoided band crossing, presence of the split-off band, and highenergy nonparabolicity all contribute to producing a temperature dependence of the hole conductivity mass that is far from simple.

To accurately model the $\mathrm{Si}$ valence bands we use a tight-binding description of the bands. ${ }^{14,16,27}$ The tight- 
binding model that we use is the three-center, orthogonal model of Papaconstantopoulos. ${ }^{14}$ This model includes up to third-neighbor interactions in an $8 \times 8$ Hamiltonian matrix that includes the $2 s$ and $2 p$ Si orbitals. As described by Chadi, ${ }^{15}$ we incorporate spin-orbit coupling of the Si $2 p$ states into the Hamiltonian, which expands the Hamiltonian to $16 \times 16$ elements. In principle, the 20 energy integrals involving the $\mathrm{Si}$ orbitals [known as Slater-Koster (SK) parameters ${ }^{27}$ ] that are the input parameters to the tight-binding theory can be directly determined theoretically. In practice, one finds them by fitting experimental or $a b$ initio calculated quantities. Commonly included in the set of fitted quantities are band energies at high-symmetry points of the Brillouin zone (BZ). Indeed, in searching the literature I found no fewer than 12 sets of three-center SK parameters that do a satisfactory job of reproducing these energies. ${ }^{14-17,28-34}$

However, our requirements for a suitable set of SK parameters are stricter than simply matching energies at high-symmetry points in the BZ. We also require an accurate description of the band curvatures at the top of the valence band at the BZ center $(k=0)$ to be able to accurately assess the low-temperature hole conductivity mass. Near the band edges at the top of the valence band the hole energies can be expressed as ${ }^{21,35}$

$$
\begin{aligned}
& E_{\mathrm{hh}}(\mathbf{k})=A k^{2} \pm\left[B^{2} k^{4}+C^{2}\left(k_{x}^{2} k_{y}^{2}+k_{y}^{2} k_{z}^{2}+k_{z}^{2} k_{x}^{2}\right)\right]^{1 / 2}, \\
& E_{\mathrm{so}}(\mathbf{k})=A k^{2}+\Delta,
\end{aligned}
$$

where hh, lh, and so indicate heavy-hole, light-hole, and split-off, respectively. The parameters $A, B$, and $C$ are known as the band-curvature parameters; $\Delta=0.044 \mathrm{eV}$, which arises from the spin-orbit interaction, is the distance of the split-off band from the heavy-hole and lighthole bands at $k=0 .{ }^{26}$ As is evident from Eqs. (9), one can easily evaluate the parameters $A,|B|$, and $|C|$ from the tight-binding band structure by calculating $\partial^{2} E_{i}(\mathbf{k}) / \partial k^{2}$ along high-symmetry directions in the BZ.

Experimentally, the curvature parameters have been inferred from cyclotron resonance and quantum spectra measurements. ${ }^{18-20,35,36}$ Table 1 lists four sets of experimentally determined values of $A,|B|$, and $|C|$. The first two sets are from cyclotron resonance measurements from two independent groups of researchers ${ }^{35,36}$; the third set, from Stickler et al., is from quantum spectra ${ }^{18}$; and

Table 1. Experimentally Derived Curvature Parameters $^{a}$

\begin{tabular}{lccc}
\hline \multicolumn{1}{c}{ Study } & $A$ & $|B|$ & $|C|$ \\
\hline Dresselhaus et al. $^{b}$ & $4.0 \pm 0.2$ & $1.1 \pm 0.5$ & $4.0 \pm 0.5$ \\
Dexter et al. ${ }^{c}$ & $4.0 \pm 0.1$ & $1.1 \pm 0.4$ & $4.1 \pm 0.4$ \\
Stickler et al. $^{d}$ & 4.22 & 1.0 & 4.34 \\
Balslev and Lawaetz & $4.27 \pm 0.02$ & $0.63 \pm 0.08$ & $4.93 \pm 0.15$ \\
(from Hensel and Feher & & & \\
\hline
\end{tabular}

${ }^{a}$ Units for the curvature parameters are $\hbar^{2} / 2 m_{0}$.

${ }^{b}$ Ref. 35 .

${ }^{c}$ Ref. 36.

${ }^{d}$ Ref. 18 .

${ }^{e}$ Ref. 20 .

${ }^{f}$ Ref. 19 .
Table 2. Curvature and SK Parameters for Three Tight-Binding Models ${ }^{a}$

\begin{tabular}{lccc}
\hline Parameter & Harrison $^{b}$ & Papaconstantopoulos $^{c}$ & Niquet et al. $^{d}$ \\
\hline$A$ & 4.26 & 3.54 & 4.26 \\
$|B|$ & 0.89 & 0.70 & 0.81 \\
$|C|$ & 4.20 & 3.69 & 4.74 \\
$E_{s s}(000)$ & -5.262 & -6.36892 & -6.15831 \\
$E_{s s}(220)$ & 0 & 0.21149 & 0.2301 \\
$E_{s x}(022)$ & 0 & 0.04306 & -0.02496 \\
$E_{s x}(220)$ & 0 & -0.1435 & -0.21608 \\
$E_{s s}(111)$ & -1.821 & -1.84407 & -1.78516 \\
$E_{s x}(111)$ & -1.301 & 1.01852 & 0.78088 \\
$E_{x x}(000)$ & 1.768 & 2.25140 & 2.41088 \\
$E_{x x}(220)$ & 0 & 0.09858 & 0.02286 \\
$E_{x x}(022)$ & 0 & -0.32894 & -0.24379 \\
$E_{x y}(220)$ & 0 & -0.12292 & -0.05462 \\
$E_{x y}(022)$ & 0 & -0.18248 & -0.12754 \\
$E_{x x}(111)$ & 0.442 & 0.29947 & 0.35657 \\
$E_{x y}(111)$ & 1.311 & 1.44623 & 1.47649 \\
$E_{s s}(311)$ & 0 & -0.11562 & -0.06857 \\
$E_{s x}(311)$ & 0 & -0.12484 & -0.25209 \\
$E_{s x}(113)$ & 0 & 0.07166 & 0.17098 \\
$E_{x x}(311)$ & 0 & 0.07920 & 0.13968 \\
$E_{x x}(113)$ & 0 & 0.02620 & -0.0458 \\
$E_{x y}(311)$ & 0 & 0.08797 & 0.03625 \\
$E_{x y}(113)$ & 0 & -0.07875 & -0.06921 \\
\hline${ }_{y}$ & 0 & &
\end{tabular}

${ }^{a}$ The SK parameters $E_{s s}(000)$ and $E_{x x}(000)$ have been uniformly shifted in each model such that the top of the valence band is at $E=0$. Units for the curvature parameters are $\hbar^{2} / 2 m_{0}$. Units for the SK parameters are electron volts.

${ }^{b}$ Ref. 16.

${ }^{c}$ Ref. 14.

${ }^{d}$ Ref. 17.

the fourth set was obtained by Balslev and Lawaetz ${ }^{20}$ from cyclotron resonance measurements on uniaxially stressed $\mathrm{Si}^{19}$ The fourth set is most often quoted in the literature. This is not surprising, given the small error bars. However, the third set, obtained with a method that is independent of the cyclotron-resonance results, is in quite good agreement with those measurements and perhaps should not be dismissed out of hand, even though it does not agree well with the fourth set. Thus I considered both the Stickler and the Balslev parameters in calculating the valence-band masses.

I evaluated the 12 sets of previously derived SK parameters ${ }^{14-17,28-34}$ to find those that produce curvature parameters closest to the experimentally derived values: three sets that produce reasonably close values were found, ${ }^{14,16,17}$ as listed in Table 2 . The first set, from the universal model of Harrison (slightly modified) ${ }^{37}$ is a nearest-neighbor model only. Thus the second- and third-neighbor SK parameters are zero. The second set, from Papaconstantopoulos, includes up to third-neighbor interactions and has been fitted to both valence- and conduction-band energies. The third SK set, from Niquet et al., ${ }^{17}$ was fitted not only to band energies but also to the Balslev curvature parameters. ${ }^{38}$ However, the Niquet valence bands do not match these curvature parameters exactly. As is shown below, the low-temperature conductivity mass is highly sensitive to the band curva- 
ture, with the Balslev and Stickler parameters producing quite different values of $m_{h}(T)$. It is therefore advantageous to be able to exactly match an experimental set of $A,|B|$, and $|C|$ with the tight-binding formalism.

I thus investigated the 20 SK parameters of the tightbinding model to ascertain how small variations in these parameters affect both the band curvature and the BZ edge energies. I found that $E_{s, x}(220), E_{x, y}(220)$, and

Table 3. Values of SK Parameters $E_{s x}(220)$, $E_{x y}(220)$, and $E_{x y}(113)$ Adjusted by Use of the Harrison, Papaconstantopoulos, and Niquet SK Parameter Sets to Match the Balslev-Stickler Curve Parameters ${ }^{a}$

\begin{tabular}{lrcc}
\hline SK Parameter & Harrison $^{b}$ & Papaconstantopoulos $^{c}$ & Niquet $^{\text {et al. }}{ }^{d}$ \\
\hline \multicolumn{2}{l}{ Balslev matched } \\
$E_{s x}(220)$ & -0.01291 & -0.16888 & -0.20354 \\
$E_{x y}(220)$ & 0.15423 & -0.05116 & -0.01312 \\
$\quad E_{x y}(113)$ & 0.01540 & -0.03798 & -0.05960 \\
Stickler matched & & \\
$E_{s x}(220)$ & 0.00459 & -0.19656 & -0.22817 \\
$E_{x y}(220)$ & 0.04162 & -0.16453 & -0.12628 \\
$E_{x y}(113)$ & -0.00860 & -0.05891 & -0.08127 \\
\hline
\end{tabular}

${ }^{a}$ See Table 2.

${ }^{b}$ Ref. 16.

${ }^{c}$ Ref. 14 .

${ }^{d}$ Ref. 17.
$E_{x, y}(113)$ produce the largest, linearly independent, relative variations in curvature compared with variations in the energies. I then adjusted $E_{s, x}(220), E_{x, y}(220)$, and $E_{x, y}(113)$ in each of the models in Table 1 to exactly match the two sets of experimental curvature parameters (Balslev and Stickler). The adjusted values of these three SK parameters are given in Table 3. The effect on the energies at the $\mathrm{BZ}$ edges with these modified SK parameter values is minimal, indicating that the hightemperature variation in conductivity mass should be insensitive to these changes in $E_{s, x}(220), E_{x, y}(220)$, and $E_{x, y}(113)$. It is shown below that such is indeed the case.

Heavy-hole, light-hole, and split-off bands along highsymmetry directions calculated with the two sets of curvature-matched Harrison parameters are shown in Fig. 3; the solid curves in Figs. 3(a) and 3(b) show bands that reproduce the Stickler and Balslev parameters, respectively. Inasmuch as $(3 / 2) k_{B} 3000 \mathrm{~K}=0.38 \mathrm{eV}$, the range of hole energies shown is appropriate for temperatures up to $\sim 3000 \mathrm{~K}$. Note the rather complicated nature of the heavy-hole and light-hole bands at hole energies below the split-off energy. Differences among bands calculated with the modified Harrison, Papaconstantopoulos, and Niquet SK parameters, when they are matched to a given set of curvature parameters, are much smaller than the differences between the two sets of curvature-matched Harrison bands shown in Fig. 3. Bands calculated with the analytic $k \cdot p$ model of Dür et $a l .{ }^{39}$ with the Balslev curvature set are shown as dotted
(100)

(110)

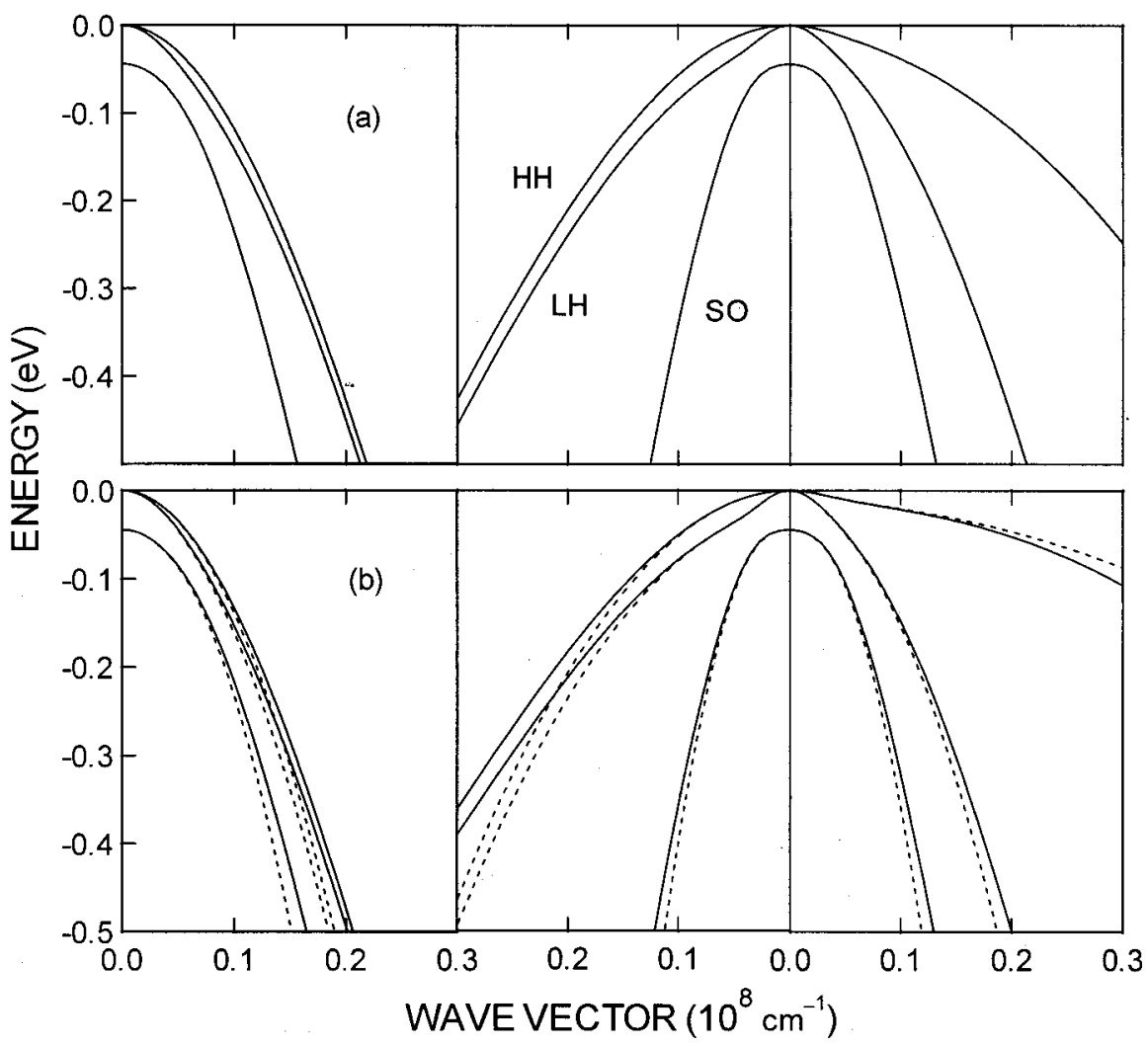

Fig. 3. Valence-band dispersion curves along high-symmetry directions near the top of the valence band. (a) Bands calculated from the Harrison model matched to the Stickler curvature parameters. (b) Solid curves, Harrison model matched to the Balslev curvature parameters; dashed curves, Dür model with the Balslev curvature parameters. 
curves in Fig. 3(b). This model includes $2 p$ spin-orbit splitting but is parabolic at high energies. Comparison of these bands with the tight-binding bands, which generally become flatter at higher energies, shows that highenergy nonparabolicity is indeed significant in all three Si valence bands.

From the tight-binding band structure the hole conductivity mass $m_{h}(T)$ is calculated from Eqs. (2) and (3) $(i$ $=\mathrm{hh}, \mathrm{lh}, \mathrm{so})$ in the limit of a Boltzmann distribution function. Typically, 330 points in the irreducible BZ are used to calculate $m_{h}(T)$. As a check on our numerical method we compare band-edge conductivity masses for each individual band (calculated at $1 \mathrm{~K}$ ) with conductivity masses calculated from the approximate expressions of Lax and Mavroides. ${ }^{40}$ For the Balslev curvature parameters we calculate heavy-hole, light-hole, and split-off masses of $0.417,0.149$, and $0.238 m_{0}$, in very good agreement with Lax-Mavroides values of $0.411,0.149$, and $0.234 m_{0}$. The results for $m_{h}(T)$ are shown in Fig. 4 . Figure 4(a) plots $m_{h}(T)$ for the six sets of curvaturematched SK parameters. The top three curves are for bands matched to the Stickler parameters, the bottom three curves correspond to the Balslev parameters. The curves show that for temperatures below $\sim 500 \mathrm{~K}$ the curvature parameters largely determine $m_{h}(T)$, whereas above $\sim 1000 \mathrm{~K}$ the high-energy nonparabolicity, which is independent of the band-edge curvature, determines the rate of change of $m_{h}(T)$ with temperature.

In conjunction with the curves in Fig. 4(a) there is experimental evidence that the Balslev parameters may be more accurate that those of Stickler. From roomtemperature reflectivity data from $p$-type Si with a carrier density of $9.6 \times 10^{18} \mathrm{~cm}^{-3}$, a conductivity mass of 0.37 $m_{0}$ has been inferred. ${ }^{10}$ This value is in excellent agreement with our room-temperature value of $m_{h}$ for the Balslev set but is significantly lower than the value of $\sim 0.41 m_{0}$ for the Stickler set.

As is clear in all the curves in Fig. 4(a), there is an initial increase in mass, followed by a slight decrease, followed again by an increase. This shape is emphasized in Fig. 4(b), which plots the temperature on a logarithmic scale. The top curve in Fig. 4(b) is an average of the three tight-binding models fitted to the Balslev curvature. To ascertain the origin of the shape of $m_{h}(T)$ we also plot masses calculated from several simpler models of the bands. The long-dashed curve in Fig. 4(b) is from the model of Dür et al. [see Fig. 3(b)], which is parabolic at high energies. ${ }^{39}$ Thus it is clear that high-energy nonparabolicity starts to become important at temperatures as low as $100 \mathrm{~K}$. As a further simplification, I also calculate $m_{h}(T)$, using the model of Dür et al. without the split-off band, shown as the short-dashed curve. The difference in the short- and long-dashed curves shows that the splitoff band becomes thermally populated only above $\sim 200 \mathrm{~K}$ and that its contribution is rather minimal, lowering the effective mass at $3000 \mathrm{~K}$ by only $\sim 0.012 \mathrm{~m}_{0}$. The dotted curve in Fig. 4(b) was calculated with the assumption that the bands maintain their zone-edge shape, given by Eq. (9), throughout the BZ. As expected, the mass is temperature independent until the split-off band begins to contribute. Thus we can identify both the initial increase and the decrease as due to the complicated shapes

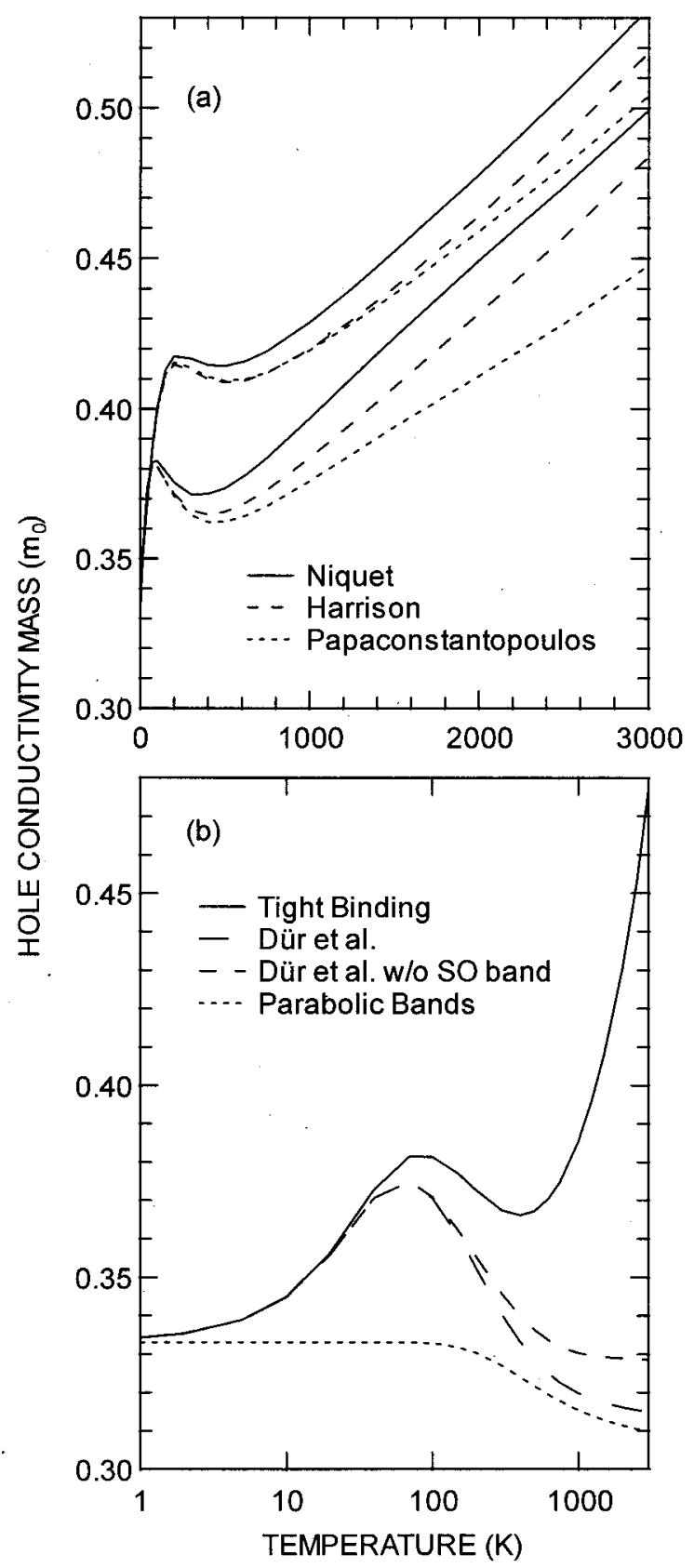

Fig. 4. Calculated hole conductivity mass versus temperature in the nondegenerate carrier-density limit. (a) Curves for the Niquet, Harrison, and Papaconstantopoulos SK parameters sets matched to the Stickler (three top curves) and Balslev (three bottom curves) curvature parameters. (b) Solid curve, average of three tight-binding calculations matched to the Balslev curvature parameters; long-dashed curve, Dür model with Balslev curvature parameters; short-dashed curve, Dür model with Balslev curvature parameters (the split-off band is neglected); dotted curve, parabolic bands, Eq. (9).

of the heavy- and light-hole bands in the energy region of the avoided crossing.

The linear nature of $m_{h}(T)$ above $\sim 500 \mathrm{~K}$ indicates that the standard phenomenological description used for the conduction band [Eq. (1)] is applicable to the valence band in this temperature range. Indeed, the average tight binding derived $m_{h}(T)$ [solid curve in Fig. 4(b)] is described to excellent approximation by use of Eq. (8) with $m_{e 0}$ replaced by $m_{h 0}=0.33 m_{0}$ and $\alpha=0.34 \mathrm{eV}^{-1}$. 


\section{COMPARISON WITH FEMTOSECOND REFLECTIVITY MEASUREMENTS}

When there is an equal number of electrons and holes, which can happen under intense laser illumination, the conductivity or optical response of the free carriers is characterized by the optical mass $m_{\text {opt }}(T)=\left[1 / m_{e}(T)\right.$ $\left.+1 / m_{h}(T)\right]^{-1}$, where carrier density $n_{c}$ is taken to be that of either the excited electrons or holes. To calculate this optical mass we use the $\alpha=0.4 \mathrm{eV}^{-1}, \beta=0.25$ $\mathrm{eV}^{-2}$ result for the electron mass and an average of the Balslev curvature-derived tight-binding hole mass. Both of these temperature-dependent masses, along with the corresponding optical mass, are plotted in Fig. 5.

This result for $m_{\text {opt }}(T)$ can be compared with our time resolved femtosecond-laser reflectivity measurements of a commercial $\mathrm{Si}(100)$ wafer. In these experiments $25-\mathrm{fs}$, 2.8-nJ pump pulses from a Ti:sapphire oscillator centered at $800 \mathrm{~nm}(1.55 \mathrm{eV})$ excite electrons from the valence to the conduction band. Electron-hole pairs with a density of $\sim 5 \times 10^{18} \mathrm{~cm}^{-3}$, which is in the nondegenerate density limit, are created. Initially, each carrier has on average $0.20 \mathrm{eV}$ of excess energy above the band minima.

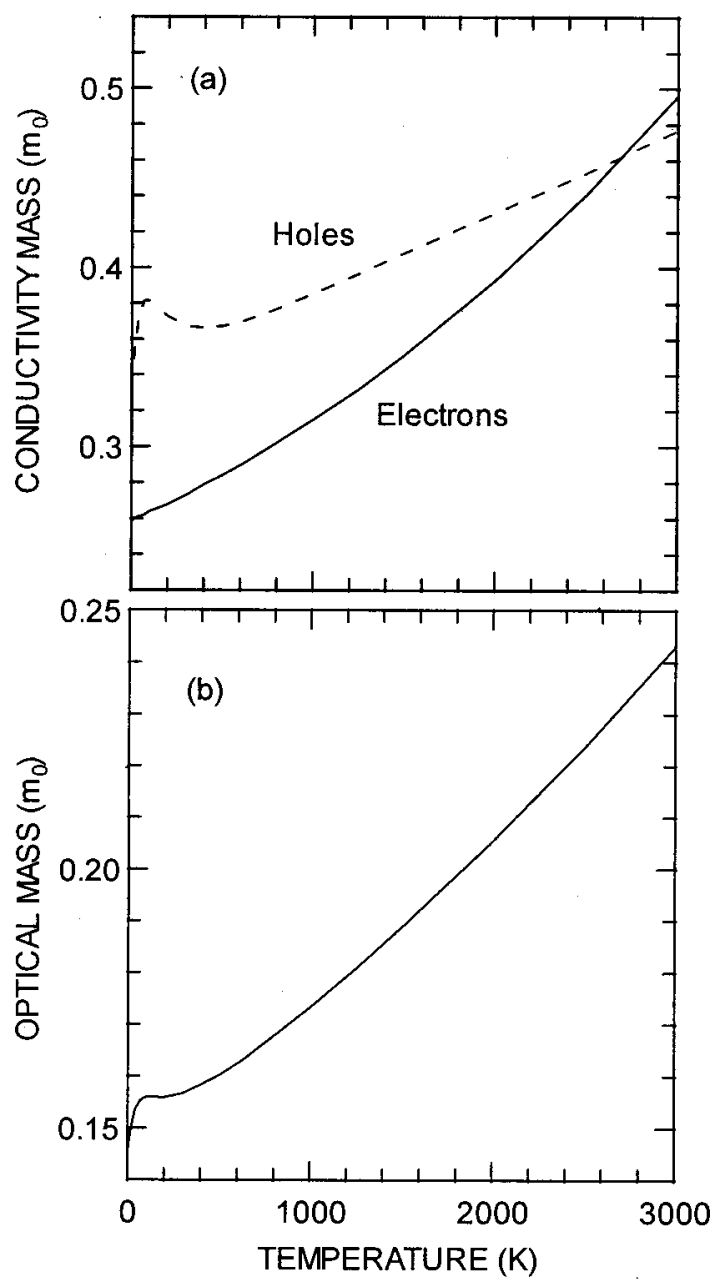

Fig. 5. Electron, hole, and optical masses versus temperature in the nondegenerate carrier density limit. (a) Electron mass from extended model with $\alpha=0.4 \mathrm{eV}^{-1}$ and $\beta=0.25 \mathrm{eV}^{-2}$. Hole mass from average of three tight-binding calculations matched to the Balslev curvature parameters. (b) Optical mass calculated from the electron and hole masses; see text.

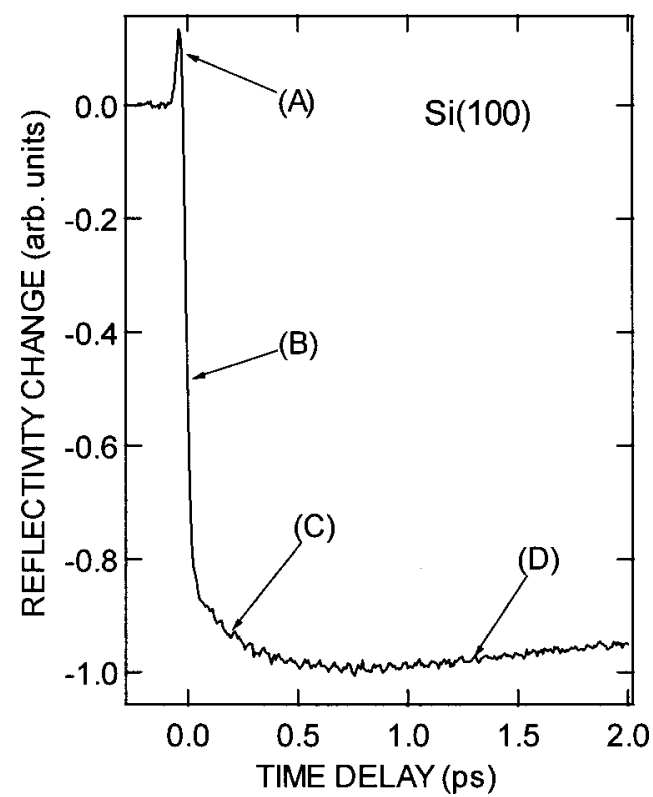

Fig. 6. Time-dependent reflectivity change of $\mathrm{Si}(100)$ after excitation by a 25 -fs, $800-n m$ laser pulse. (A) Coherent spike, (B) pulse-width-limited drop in reflectivity caused by optical response of excited free carriers, (C) continued drop caused by decrease in optical mass as carriers cool to room temperature, (D) beginning of increase in reflectivity owing to carrier recombination across the $\mathrm{Si}$ bandgap.

Within a few tens of femtoseconds this excess energy is thermalized among the carriers at a temperature of 1550 $\mathrm{K}{ }^{41,42}$ The carriers then equilibrate with the lattice with a time constant of approximately $200-400 \mathrm{fs},{ }^{8,41-44}$ which cools them to nearly room temperature because the electronic specific heat is much less than the lattice specific heat. On much longer time scales, typically $>1 \mu \mathrm{s}$, the electrons and holes recombine across the band gap. ${ }^{21}$

The electron dynamics of the laser pulse-excited $\mathrm{Si}$ sample are followed in real time by measurement of the change in reflectivity with 25 -fs, 100 -pJ probe pulses. Figure 6 shows typical reflectivity data. After a small initial spike that is due to coherent effects between the pump and the probe pulses [part (A) of the reflectivity curve], the reflectivity exhibits a relatively large laser pulse width-limited decrease (B), followed by a smaller, continuing decrease on a much slower time scale $(\mathrm{C})$. At $\sim 1$ ps the reflectivity begins to increase (D). (The small oscillations in the reflectivity are due to coherent excitation of the Si zone-center optic phonon, analogous to those observed in ultrafast reflectivity experiments with $\mathrm{Ge} .{ }^{45}$ ) The sharp drop in reflectivity $R$ at (B) is due mainly to the free-carrier optical response of the laser generated electron-hole pairs. ${ }^{46}$ Using the standard Drude model of optical conductivity, ${ }^{24}$ which accurately describes the free-carrier response in $\mathrm{Si}^{10-13}$ we can show that the reflectivity decrease that is due to the free carriers can be written as

$$
\frac{\Delta R}{R}=-\gamma \frac{n_{c}}{m_{\mathrm{opt}}},
$$

where $\gamma$ is a parameter that depends on the probe-pulse angle of incidence, laser frequency, and $\mathrm{Si}$ index of refraction. ${ }^{47,48}$ The continuing slower decrease at (C), 
which occurs with a relaxation time constant of 253 $\pm 7 \mathrm{fs}$, is attributed to the decrease in $m_{\text {opt }}$ as the electrons and holes cool from $1550 \mathrm{~K}$ to room temperature. Note that this relaxation time is in excellent agreement with a recent determination of the carrier energyrelaxation time of $240 \pm 30 \mathrm{fs}$ found from transient grating spectroscopy. ${ }^{8}$ From the measurements reported here, the magnitude of this slower drop in reflectivity yields a change in $m_{\text {opt }}$ of $22.9 \pm 0.8 \%$ from 300 to 1550 $\mathrm{K}$, in excellent agreement with the value of $21.9 \%$ deduced from the theoretical curve in Fig. 5, thus confirming the interpretation of the femtosecond reflectivity data.

\section{CONCLUSIONS AND SUMMARY}

An overall comparison of calculated electron, hole, and optical masses with the two earlier calculations ${ }^{6,7}$ is given in Table 4. At $300 \mathrm{~K}$ all three calculations are in reasonable agreement for all three masses, with the best agreement between the present calculations and the pseudopotential band-structure-based results of van Driel. ${ }^{6}$ At $1700 \mathrm{~K}$ the present results also agree quite well with those of Yang and Bloembergen. ${ }^{7}$ At $3000 \mathrm{~K}$ our electron conductivity mass again is reasonably close to that of van Driel. We note, however, that the standard conduction-band model with $\alpha=0.6 \mathrm{eV}^{-1}$ (dashed curve in Fig. 2) is in even better agreement with van Driel's result. However, the 3000-K hole masses are quite different: we obtain $0.48 m_{0}$, compared with $0.87 m_{0}$ by van Driel. It thus appears that the high-energy nonparabolicity in the valence bands used by van Driel is approximately twice as strong as in any of our tight-binding bands.

Although our aim has been to describe accurately the electron and hole effective masses, these calculations, especially the tight-binding parameterizations presented here, may be useful to researchers interested in other properties of Si. For example, it has been noted that tight-binding parameters that accurately reproduce the band-edge curvature are essential for describing the electronic states of Si nanostructures. ${ }^{17}$ An accurate description of the bands, both near the band edge and at higher energies, is also essential for Monte Carlo simulations of high-field transport in semiconductor devices. ${ }^{1,49}$

Table 4. Comparison of Calculated Values of $m_{e}(T), m_{h}(T)$, and $m_{\text {opt }}(T)$ among Present Study, van Driel Study, and Yang-Bloembergen Study ${ }^{a}$

\begin{tabular}{lcccc}
\hline \multicolumn{1}{c}{ Study } & Temperature $(\mathrm{K})$ & $m_{e}(T)$ & $m_{h}(T)$ & $m_{\text {opt }}(T)$ \\
\hline Present & 300 & 0.273 & 0.367 & 0.156 \\
& 1700 & 0.367 & 0.418 & 0.195 \\
& 3000 & 0.496 & 0.477 & 0.243 \\
Yang et al. $^{b}$ & 300 & 0.291 & 0.397 & 0.168 \\
& 1700 & 0.388 & 0.435 & 0.205 \\
Van Driel $^{\mathrm{b}}$ & 300 & 0.272 & 0.373 & 0.157 \\
& 3000 & 0.447 & 0.872 & 0.295 \\
\hline
\end{tabular}

${ }^{a}$ The masses are in units of $\mathrm{m}_{0}$.

${ }^{b}$ Ref. 7.

${ }^{c}$ Ref. 6.
In summary, the temperature-dependent electron and hole conductivity masses in $\mathrm{Si}$ in the limit of nondegenerate carrier densities have been calculated. A standard conduction-band nonparabolicity model was used that was evaluated against reflectivity-derived densitydependent electron masses, yielding a nonparabolicity parameter of $0.6 \mathrm{eV}^{-1}$. A slightly better description of the density-dependent masses was obtained by extension of the standard nonparabolicity model to the next higher term in energy. Tight-binding calculated valence bands have been used in evaluation of the hole conductivity mass, which has a much richer structure relative to temperature than do the electrons. The complexity of relating $m_{h}$ to $T$ is most apparent below $500 \mathrm{~K}$ and is due mainly to the structure in the heavy- and light-hole bands below the split-off band energy. Above $500 \mathrm{~K}$ the standard conduction band model can be used for the holes with a nonparabolicity parameter of $0.34 \mathrm{eV}^{-1}$. The resulting calculated change in the optical mass from 300 to $1550 \mathrm{~K}$ compares extremely well with results from timeresolved femtosecond reflectivity measurements.

\section{REFERENCES AND NOTES}

1. C. Jacoboni and L. Reggiani, "The Monte Carlo method for the solution of charge transport in semiconductors with application to covalent materials," Rev. Mod. Phys. 55, 645705 (1983).

2. L.-A. Lompre, J.-M. Liu, H. Kurz, and N. Bloembergen, "Optical heating of electron-hole plasma in silicon by picosecond pulses," Appl. Phys. Lett. 44, 3-5 (1984).

3. J.-M. Liu, H. Kurz, and N. Bloembergen, "Picosecond timeresolved plasma and temperature-induced changes of reflectivity and transmission in silicon" Appl. Phys. Lett. 41, 643-646 (1982).

4. D. von der Linde and N. Fabricius, "Observation of an electronic plasma in picosecond laser annealing of silicon," Appl. Phys. Lett. 41, 991-993 (1982).

5. C. V. Shank, R. Yen, and C. Hirlmann, "Time-resolved reflectivity measurements of femtosecond-optical-pulseinduced phase transitions in silicon," Phys. Rev. Lett. 50, 454-457 (1983).

6. H. M. van Driel, "Optical effective mass of high density carriers in silicon,” Appl. Phys. Lett. 44, 617-619 (1984).

7. G.-Z. Yang and N. Bloembergen, "Effective mass in picosecond laser-produced high-density plasma in silicon," IEEE J. Quantum Electron. QE-22, 195-196 (1986).

8. T. Sjodin, H. Petek, and H.-L. Dai, "Ultrafast carrier dynamics in silicon: a two-color transient-reflection grating study on a (111) surface," Phys. Rev. Lett. 81, 5664-5667 (1998).

9. E. M. Conwell and M. O. Vassell, "High-field transport in $n$-type GaAs," Phys. Rev. 166, 797-821 (1968).

10. W. G. Spitzer and H. Y. Fan, "Determination of optical constants and carrier effective mass of semiconductors," Phys. Rev. 106, 882-890 (1957).

11. L. E. Howarth and J. F. Gilbert, "Determination of free electron effective mass of $n$-type silicon," J. Appl. Phys. 34, 236-237 (1963).

12. M. Miyao, T. Motooka, N. Natsuaki, and T. Tokuyama, "Change in the electron effective mass in extremely heavily doped $n$-type Si obtained by ion implantation and laser annealing," Solid State Commun. 37, 605-608 (1981).

13. A. Borghesi, A. Stella, P. Bottazzi, G. Guizzetti, and L. Reggiani, "Optical determination of Si conduction-band nonparabolicity,” J. Appl. Phys. 67, 3102-3106 (1990).

14. D. A. Papaconstantopoulos, Handbook of the Band Structure of Elemental Solids (Plenum, New York, 1986).

15. D. J. Chadi, "Spin-orbit splitting in crystalline and compo- 
sitionally disordered semiconductors," Phys. Rev. B 16, 790-796 (1977).

16. W. A. Harrison, Electronic Structure and the Properties of Solids: The Physics of the Chemical Bond (Dover, New York, 1989).

17. Y. M. Niquet, C. Delerue, G. Allan, and M. Lannoo, "Method for tight-binding parameterization: application to silicon nanostructures," Phys. Rev. 62, 5109-5116 (2000).

18. J. J. Stickler, H. J. Zeigler, and G. S. Heller, "Quantum effects in Ge and Si. I," Phys. Rev. 127, 1077-1084 (1962).

19. J. C. Hensel and G. Feher, "Cyclotron resonance experiments in uniaxially stressed silicon: valence band inverse mass parameters and deformation potentials," Phys. Rev. 129, 1041-1062 (1963).

20. I. Balslev and P. Lawaetz, "On the interpretation of the observed hole mass shift with uniaxial stress in silicon," Phys. Lett. 19, 6-7 (1965).

21. K. Seeger, Semiconductor Physics: An Introduction (Springer, New York, 1982).

22. C. Jacoboni, R. Minder, and G. Majni, "Effects of band nonparabolocity on electron drift velocity in silicon above room temperature," J. Chem. Phys. Solids 36, 1129-1133 (1975).

23. G. N. Koskowich, M. Soma, and R. B. Darling, "Nearinfrared free-carrier optical absorption in silicon: effect of first-order phonon-assisted scattering in a nonparabolic conduction band," Phys. Rev. B 41, 2944-2947 (1990).

24. F. Wooten, Optical Properties of Solids (Academic, New York, 1972)

25. N. W. Ashcroft and N. D. Mermin, Solid State Physics (Saunders, Philadelphia, Pa., 1976).

26. O. Madelung, Semiconductors—Basic Data (Springer, New York, 1996)

27. J. C. Slater and G. F. Koster, "Simplified LCAO method for the periodic potential problem," Phys. Rev. 94, 1498-1524 (1954).

28. D. J. Chadi and M. L. Cohen, "Tight-binding calculations of the valence bands of diamond and zincblende crystals," Phys. Status Solidi B 68, 405-419 (1975).

29. K. C. Pandey and J. C. Phillips, "Atomic densities of states near Si(111) surfaces," Phys. Rev. B 13, 750-760 (1976).

30. D. A. Papaconstantopoulos and E. N. Economou, "SlaterKoster parameterization for Si and the ideal-vacancy calculation," Phys. Rev. B 22, 2903-2907 (1980).

31. D. N. Talwar and C. S. Ting, "Tight-binding calculations for the electronic structure of isolated vacancies and impurities in III-V compound semiconductors," Phys. Rev. B 25, 2660 2680 (1982)

32. Y. Li and P. J. Lin-Chung, "New semiempirical construction of the Slater-Koster parameters for group-IV semiconductors," Phys. Rev. B 27, 3465-3470 (1983).

33. C. Tserbak, H. M. Polatoglou, and G. Theodorou, "Unified approach to the electronic structure of strained $\mathrm{Si} / \mathrm{Ge}$ superlattices," Phys. Rev. B 47, 7104-7124 (1993).
34. G. Grosso and C. Piermarocchi, "Tight-binding model and interaction scaling laws for silicon and germanium," Phys. Rev. B 51, 16772-16777 (1995).

35. G. Dresselhaus, A. F. Kip, and C. Kittel, "Cyclotron resonance of electrons and holes in silicon and germanium crystals," Phys. Rev. 98, 368-384 (1955).

36. R. N. Dexter, H. J. Zeigler, and B. Lax, "Cyclotron resonance experiments in silicon and germanium," Phys. Rev. 104, 637-664 (1956).

37. The universal model of Harrison ${ }^{16}$ has $E_{s x}(111)=1.131$. Increasing it by $15 \%$ to 1.301 produces much better curvature parameters and BZ edge band energies.

38. The reported Niquet SK parameters $E_{s x}(311), E_{s x}(113)$, $E_{x y}(311)$, and $E_{x y}(113)$ have signs opposite those of the convention of Papaconstantopoulos. The Papaconstantopoulos convention is used in Table 2 .

39. M. Dür, K. Unterrainer, and E. Gornik, "Effect of valenceband anisotropy and nonparabolicity on total scattering rates for holes in nonpolar semiconductors," Phys. Rev. B 49, 13991-13994 (1994).

40. B. Lax and J. G. Mavroides, "Statistics and galvanomagnetic effects in germanium and silicon with warped energy surfaces," Phys. Rev. 100, 1650-1657 (1955).

41. J. R. Goldman and J. A. Prybyla, "Ultrafast dynamics of laser-excited electron distributions in silicon," Phys. Rev. Lett. 72, 1364-1367 (1994).

42. S. Jeong and J. Bokor, "Ultrafast carrier dynamics near the Si(100) 2 × 1 surface," Phys. Rev. B 59, 4943-4951 (1999).

43. F. E. Doany and D. E. Grischkowsky, "Measurement of ultrafast hot-carrier relaxation in silicon by thin film enhanced, time-resolved reflectivity," Appl. Phys. Lett. 52, 36-38 (1988).

44. W. Kütt, A. Esser, K. Seibert, U. Lemmer, and H. Kurz, "Femtosecond studies of plasma formation in crystalline and amorphous silicon," in Applications of Ultrashort Laser Pulses in Science and Technology, A. Antonetti, ed., Proc. SPIE 1268, 154-165 (1990).

45. T. Pfeifer, W. Kütt, H. Kurz, and R. Scholz, "Generation and detection of coherent optical phonons in germanium," Phys. Rev. Lett. 69, 3248-3251 (1992).

46. A. J. Sabbah and D. M. Riffe, "Measurement of silicon surface recombination velocity using ultrafast pump-probe reflectivity in the near infrared," J. Appl. Phys. 88, 69546956 (2000).

47. O. B. Wright and V. E. Gusev, "Acoustic generation in crystalline silicon with femtosecond optical pulses," Appl. Phys. Lett. 66, 1190-1192 (1995).

48. T. Tanaka, A. Harata, and T. Sawada, "Subpicosecond surface-restricted carrier and thermal dynamics by transient reflectivity measurements," J. Appl. Phys. 82, 40334038 (1997).

49. S. M. Sze, Physics of Semiconductor Devices (Wiley, New York, 1981) 